\title{
Regression analysis of additive hazards model with latent variables
}

\author{
Supplementary Material
}


Table S1: Results of Simulation 1 - Type (I) $\lambda_{0}(t), n=500, \mathbf{B}$ is overlapping

\begin{tabular}{|c|c|c|c|c|c|c|}
\hline Model & $\mathrm{CR}$ & Para & Bias & $\mathrm{SE}$ & SEE & $\mathrm{CP}$ \\
\hline \multirow[t]{6}{*}{ AH model } & \multirow[t]{3}{*}{$20 \%$} & $\beta$ & -0.005 & 0.366 & 0.350 & 0.937 \\
\hline & & $\gamma_{1}$ & -0.007 & 0.192 & 0.192 & 0.953 \\
\hline & & $\gamma_{2}$ & 0.007 & 0.190 & 0.192 & 0.952 \\
\hline & \multirow[t]{3}{*}{$50 \%$} & $\beta$ & -0.004 & 0.449 & 0.443 & 0.938 \\
\hline & & $\gamma_{1}$ & 0.003 & 0.249 & 0.247 & 0.945 \\
\hline & & $\gamma_{2}$ & 0.012 & 0.250 & 0.248 & 0.955 \\
\hline \multirow[t]{15}{*}{ CFA model } & & $b_{11}$ & -0.001 & 0.042 & 0.043 & 0.958 \\
\hline & & $b_{21}$ & 0.001 & 0.042 & 0.043 & 0.967 \\
\hline & & $b_{31}$ & 0.000 & 0.038 & 0.038 & 0.948 \\
\hline & & $b_{41}$ & 0.001 & 0.038 & 0.038 & 0.949 \\
\hline & & $b_{12}$ & -0.000 & 0.043 & 0.043 & 0.953 \\
\hline & & $b_{42}$ & 0.002 & 0.042 & 0.043 & 0.956 \\
\hline & & $b_{52}$ & 0.001 & 0.037 & 0.038 & 0.954 \\
\hline & & $b_{62}$ & 0.001 & 0.038 & 0.038 & 0.944 \\
\hline & & $\phi_{12}$ & -0.000 & 0.052 & 0.053 & 0.953 \\
\hline & & $\psi_{1}$ & -0.001 & 0.030 & 0.029 & 0.938 \\
\hline & & $\psi_{2}$ & -0.001 & 0.028 & 0.028 & 0.947 \\
\hline & & $\psi_{3}$ & -0.002 & 0.028 & 0.028 & 0.951 \\
\hline & & $\psi_{4}$ & -0.002 & 0.030 & 0.029 & 0.939 \\
\hline & & $\psi_{5}$ & 0.000 & 0.029 & 0.028 & 0.951 \\
\hline & & $\psi_{6}$ & -0.001 & 0.028 & 0.028 & 0.952 \\
\hline
\end{tabular}


Table S2: Results of Simulation 1 - Type (I) $\lambda_{0}(t), n=500, \mathbf{B}$ is misspecified

\begin{tabular}{|c|c|c|c|c|c|c|c|c|c|c|}
\hline \multicolumn{5}{|c|}{ CFA model } & \multicolumn{6}{|c|}{ AH model } \\
\hline Para. & Bias & SE & SEE & $\mathrm{CP}$ & CR & Para. & Bias & SE & SEE & $\mathrm{CP}$ \\
\hline$b_{11}$ & -0.026 & 0.041 & 0.058 & 0.969 & $20 \%$ & $\beta_{1}$ & 0.000 & 0.008 & 0.007 & 0.946 \\
\hline$b_{21}$ & 0.020 & 0.043 & 0.055 & 0.974 & & $\beta_{2}$ & 0.000 & 0.007 & 0.007 & 0.947 \\
\hline$b_{32}$ & 0.002 & 0.075 & 0.084 & 0.973 & & $\beta_{3}$ & 0.001 & 0.016 & 0.015 & 0.936 \\
\hline$b_{42}$ & -0.000 & 0.080 & 0.095 & 0.967 & & $\gamma_{1}$ & -0.000 & 0.009 & 0.009 & 0.939 \\
\hline$b_{53}$ & 0.027 & 0.066 & 0.115 & 0.975 & & $\gamma_{2}$ & -0.001 & 0.009 & 0.009 & 0.944 \\
\hline$b_{63}$ & -0.031 & 0.070 & 0.138 & 0.965 & & $\gamma_{3}$ & -0.000 & 0.008 & 0.008 & 0.947 \\
\hline$b_{74}$ & 0.033 & 0.060 & 0.081 & 0.873 & & $\gamma_{4}$ & 0.001 & 0.010 & 0.010 & 0.965 \\
\hline$b_{84}$ & -0.123 & 0.101 & 0.093 & 0.556 & $50 \%$ & $\beta_{1}$ & -0.000 & 0.010 & 0.009 & 0.941 \\
\hline \multirow[t]{6}{*}{$b_{94}$} & -0.137 & 0.144 & 0.196 & 0.563 & & $\beta_{2}$ & -0.000 & 0.010 & 0.009 & 0.953 \\
\hline & & & & & & $\beta_{3}$ & 0.001 & 0.020 & 0.019 & 0.950 \\
\hline & & & & & & $\gamma_{1}$ & 0.000 & 0.011 & 0.011 & 0.942 \\
\hline & & & & & & $\gamma_{2}$ & -0.001 & 0.012 & 0.011 & 0.947 \\
\hline & & & & & & $\gamma_{3}$ & -0.000 & 0.011 & 0.011 & 0.957 \\
\hline & & & & & & $\gamma_{4}$ & 0.001 & 0.012 & 0.012 & 0.975 \\
\hline
\end{tabular}


Table S3: Results of Simulation 1 - Type (I) $\lambda_{0}(t), n=500, \boldsymbol{\epsilon}_{i} \sim N(\mathbf{0}, \mathbf{I})$

\begin{tabular}{|c|c|c|c|c|c|c|}
\hline Model & CR & Para & Bias & $\mathrm{SE}$ & SEE & $\mathrm{CP}$ \\
\hline \multirow[t]{6}{*}{ AH model } & $20 \%$ & $\beta$ & 0.015 & 0.361 & 0.355 & 0.943 \\
\hline & & $\gamma_{1}$ & 0.001 & 0.233 & 0.224 & 0.953 \\
\hline & & $\gamma_{2}$ & 0.010 & 0.227 & 0.225 & 0.943 \\
\hline & $50 \%$ & $\beta$ & 0.009 & 0.436 & 0.447 & 0.955 \\
\hline & & $\gamma_{1}$ & -0.002 & 0.290 & 0.287 & 0.963 \\
\hline & & $\gamma_{2}$ & 0.016 & 0.286 & 0.287 & 0.958 \\
\hline \multirow[t]{13}{*}{ CFA model } & & $b_{11}$ & 0.001 & 0.071 & 0.070 & 0.947 \\
\hline & & $b_{21}$ & 0.001 & 0.071 & 0.070 & 0.946 \\
\hline & & $b_{31}$ & -0.000 & 0.070 & 0.070 & 0.950 \\
\hline & & $b_{42}$ & 0.001 & 0.071 & 0.070 & 0.949 \\
\hline & & $b_{52}$ & -0.003 & 0.070 & 0.070 & 0.955 \\
\hline & & $b_{62}$ & -0.001 & 0.068 & 0.070 & 0.958 \\
\hline & & $\phi_{12}$ & 0.001 & 0.067 & 0.066 & 0.948 \\
\hline & & $\psi_{1}$ & -0.005 & 0.101 & 0.100 & 0.950 \\
\hline & & $\psi_{2}$ & -0.005 & 0.099 & 0.100 & 0.953 \\
\hline & & $\psi_{3}$ & -0.006 & 0.100 & 0.100 & 0.948 \\
\hline & & $\psi_{4}$ & -0.007 & 0.100 & 0.100 & 0.956 \\
\hline & & $\psi_{5}$ & -0.004 & 0.100 & 0.100 & 0.947 \\
\hline & & $\psi_{6}$ & -0.008 & 0.095 & 0.100 & 0.959 \\
\hline
\end{tabular}




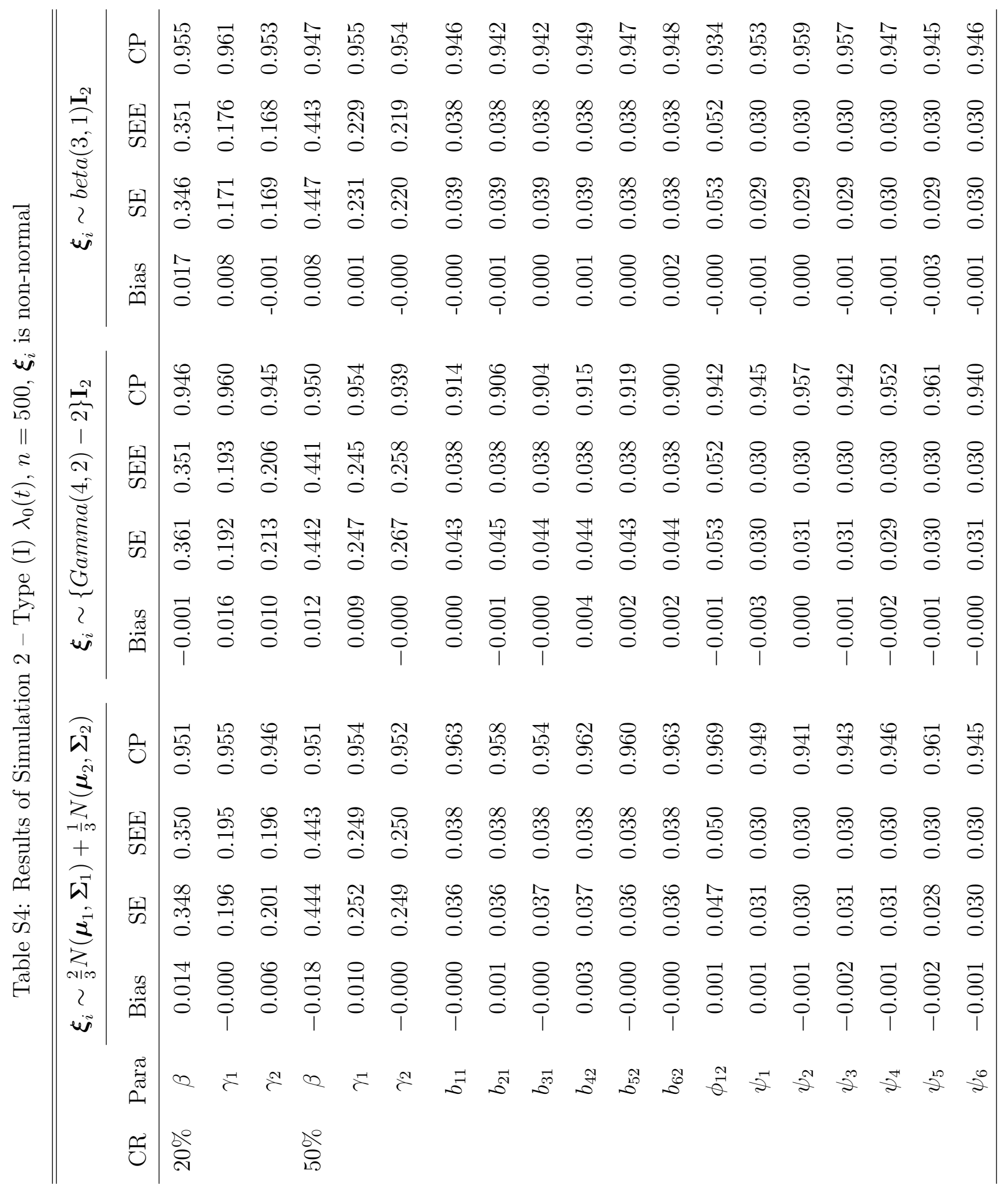




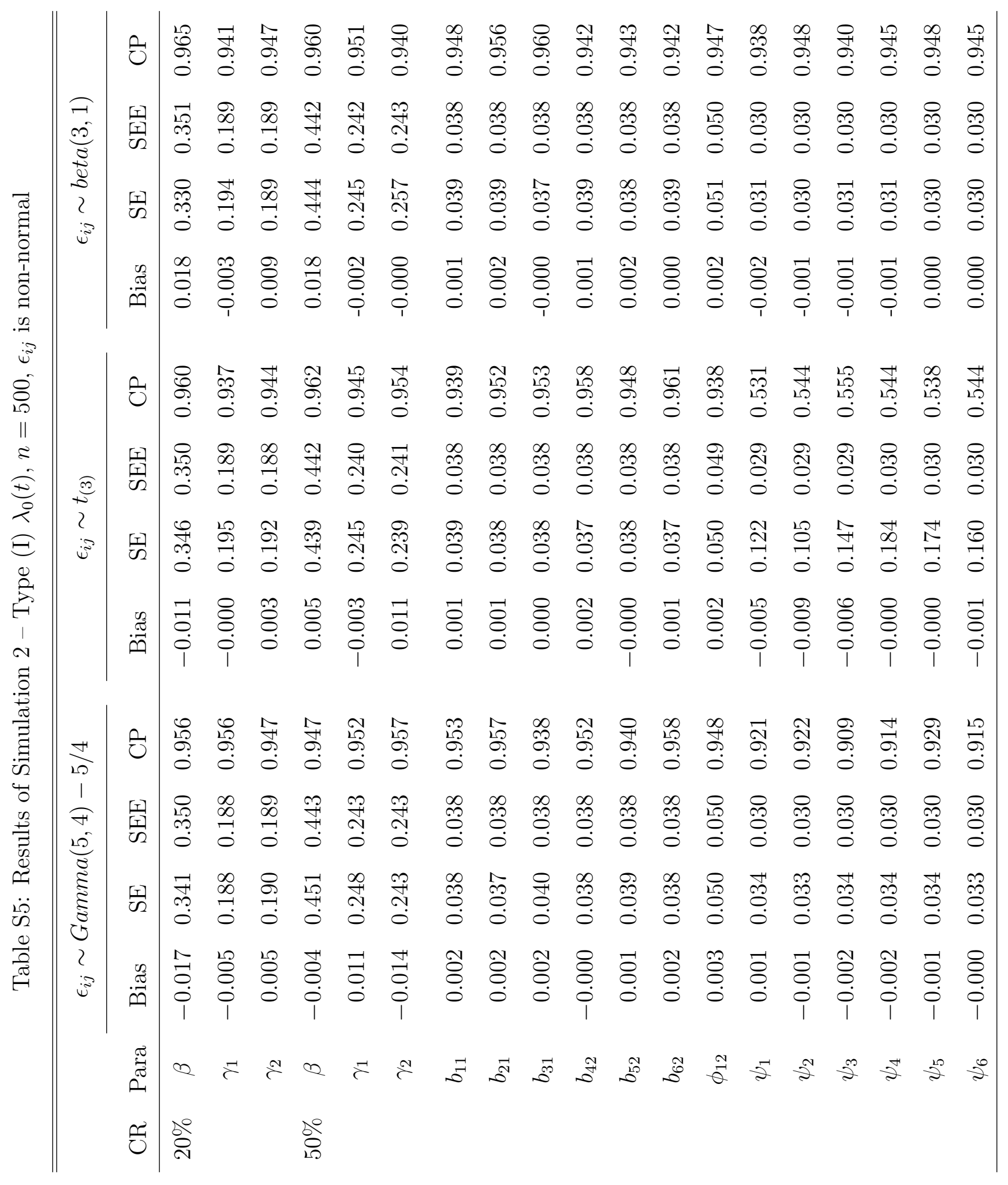


Table S6: Comparison of the CKD data analysis under different structures of $\mathbf{B}$

\begin{tabular}{|c|c|c|c|c|c|}
\hline \multirow[b]{2}{*}{ Model } & \multirow[b]{2}{*}{ Variable } & \multicolumn{2}{|c|}{$\begin{array}{c}\text { B is overlapping } \\
\quad b_{81} \neq 0\end{array}$} & \multicolumn{2}{|c|}{$\begin{array}{c}\mathbf{B} \text { is nonoverlapping } \\
b_{81}=0\end{array}$} \\
\hline & & Est & SEE & Est & SEE \\
\hline \multirow[t]{10}{*}{ CFA } & $b_{11}$ & 0.9899 & 0.0192 & 0.9551 & 0.0240 \\
\hline & $b_{21}$ & 0.8347 & 0.0184 & 0.8654 & 0.0229 \\
\hline & $b_{81}$ & -0.2490 & 0.0163 & - & - \\
\hline & $b_{32}$ & 0.7266 & 0.0312 & 0.7143 & 0.0311 \\
\hline & $b_{42}$ & 0.8464 & 0.0350 & 0.8611 & 0.0358 \\
\hline & $b_{53}$ & 0.7261 & 0.0387 & 0.7331 & 0.0377 \\
\hline & $b_{63}$ & 0.9457 & 0.0485 & 0.9368 & 0.0463 \\
\hline & $b_{74}$ & 0.3395 & 0.0192 & 0.3413 & 0.0193 \\
\hline & $b_{84}$ & -0.2535 & 0.0163 & -0.3042 & 0.0185 \\
\hline & $b_{94}$ & 0.9925 & 0.0437 & 0.9834 & 0.0484 \\
\hline \multirow[t]{7}{*}{$\mathrm{AH}$} & Age at enrollment & 0.0188 & 0.0013 & 0.0191 & 0.0013 \\
\hline & Duration of diabetes & 0.0140 & 0.0017 & 0.0140 & 0.0017 \\
\hline & Sex & -0.0030 & 0.0022 & -0.0032 & 0.0022 \\
\hline & Obesity & 0.0029 & 0.0012 & 0.0028 & 0.0013 \\
\hline & Blood pressure & 0.0042 & 0.0016 & 0.0038 & 0.0016 \\
\hline & Glycemia & 0.0038 & 0.0015 & 0.0040 & 0.0015 \\
\hline & Lipid & 0.0069 & 0.0017 & 0.0071 & 0.0017 \\
\hline
\end{tabular}

